\title{
Magnetic Resonance Elastography
}

National Cancer Institute

\section{Source}

National Cancer Institute. Magnetic Resonance Elastography. NCI Thesaurus. Code C19338.

This imaging technique returns information about the mechanical properties (feel) of tissues by combining MRI with ultrasound. A properly equipped MRI machine captures a cross sectional image of the sound waves, produced by ultrasound techniques, which are then processed by computer to produce an image showing the hardness or elasticity of tissues within the cross section. 